\title{
Haptic dancing: human performance at haptic decoding with a vocabulary*
}

\author{
Sommer Gentry \\ Lab for Information and Decision Systems \\ Massachusetts Institute of Technology \\ Cambridge, Massachusetts, U. S. A. \\ sommerg@mit.edu
}

\author{
Roderick Murray-Smith \\ Hamilton Institute \\ National University of Ireland, Maynooth \\ and Department of Computing Science \\ University of Glasgow \\ Glasgow, Scotland, United Kingdom \\ rod@dcs.gla.ac.uk
}

\begin{abstract}
The inspiration for this study is the observation that swing dancing involves coordination of actions between two humans that can be accomplished by pure haptic signaling. This study implements a leaderfollower dance to be executed between a human and a PHANToM haptic device. The data demonstrate that the participants' understanding of the motion as a random sequence of known moves informs their following, making this vocabulary-based interaction fundamentally different from closed loop pursuit tracking. This robot leader does not respond to the follower's movement other than to display error from a nominal path. This work is the first step in an investigation of the successful haptic coordination between dancers, which will inform a subsequent design of a truly interactive robot leader.
\end{abstract}

Keywords: Haptics, control, haptic interaction, rhythmic interaction, pursuit tracking, precognitive tracking, coordination, motion primitives, haptic communication.

\section{Overview and Background}

Swing dancing requires coordination of actions between two humans by pure haptic signaling. Previous work on haptic interfaces between humans found evidence that haptic-only cooperation was inferior to visual-only cooperation and found no evidence that haptic-plusvisual cooperation was superior to visual-only communication of a Japanese calligraphic character between users unacquainted with these characters [8]. However, experienced swing dance followers have demonstrated in our lab the ability to correctly identify moves while deprived by blindfold of visual information. One distinction between these two tasks is use of a shared

${ }^{*}$ 0-7803-7952-7/03/\$17.00 (C) 2003 IEEE. vocabulary of primitive moves in swing dance versus continuous force error nulling in the Japanese character study.

The primary goal in this study is to investigate the participant's interaction strategies in the specific context of improvised dance, hence the experiment was designed to be faithful to the dancing model.

A brief review of a number of classical results in control system modeling of human operators can be found in Hess [5]. These results, such as McRuer's frequency domain crossover model, are formulated for random or random-appearing inputs or disturbances. The task of following a sequence of dance moves from a known vocabulary does not fit this model, because the dance moves are idealized and known a priori, and transitions between moves are constrained to happen at specific times during a dance. Move transitions land on the beat of the music that both partners hear.

More closely related to the current study are experiments such as those of Jagacinski [6]. His subjects repeatedly tracked identical 20 second segments with a position control joystick via visual display of error. With practice, subjects were able to reduce the effective delay of their responses from about $126 \mathrm{~ms}$ to about $32 \mathrm{~ms}$. Effective delay was calculated as the best fit $\tau$ in a Taylor-series approximation to an input reconstruction model $x(t)=\beta_{0}+I(t-\tau)$, where $x(t)$ is the subject's position and $I(t-\tau)$ is the input $\tau$ seconds prior. As $32 \mathrm{~ms}$ delays in visuomotor loops are physiologically infeasible, this effective delay reflects learning of the input signal $I$.

Then, the current experiments can be seen as exploring the space between exactly repeated tracking tasks and apparently random input tracking tasks. In this work, as in extemporaneous partner dance, repeated moves are sequenced in novel or in predictable ways. 


\subsection{Successive organizations of percep- tion}

The successive organizations of perception theory of humans as controllers described three levels of behavior: compensatory, pursuit, and precognitive [7]. In compensatory tracking, the subject controls the perceived error. In pursuit tracking, the subject observes both perceived error and perceived or estimated target. Even if, as in this experiment, the target is not displayed, experienced subjects estimate it from the displayed error and behave as if it were present. In precognitive tracking, the subject chooses a pattern, known or assumed, for the target and responds in a zero-delay preprogrammed, i.e. open loop, fashion.

The question this study asks is whether the subjects can use precognitive controls when confronted with repeated moves sequenced unpredictably.

\subsection{Haptic modality}

Classical research on human tracking ability considered mostly tracking visual displays of error using a joystick. The presentation of error here is novel. While the subjects can see the path of the PHANToM's stylus, they can only perceive error levels haptically, interpreting greater forces as greater errors. Just noticeable differences are larger for haptic signals than for visual ones, so it is to be expected that mean squared tracking error, for instance, is higher in this haptic-display movement task [1].

\subsection{Paper outline}

In the remainder of the paper: first, the experimental setup and protocol are reviewed briefly. The design of metrics for the task and results of the experiments are presented, and conclusions follow.

\section{Experiments}

The PHANToM, a desktop haptic device, presents a force at its stylus endpoint which is a function of its sensed position. This study used the PHANToM to implement a dance game with lead and follow maneuvers. A more detailed account of the system design is given in Gentry et al. [4].

The PHANToM leads a human follower in unknown sequences of known moves. The device acts as a modified proportional-derivative feedback controller to lead the human user. The patterns are two dimensional in $x$ and $y$, with $z$ reserved for a study of connection. The force imposed, $F$, is a function of the error signal $e_{p}$ and $e_{v}$ in $x$ and $y$, the difference of position and

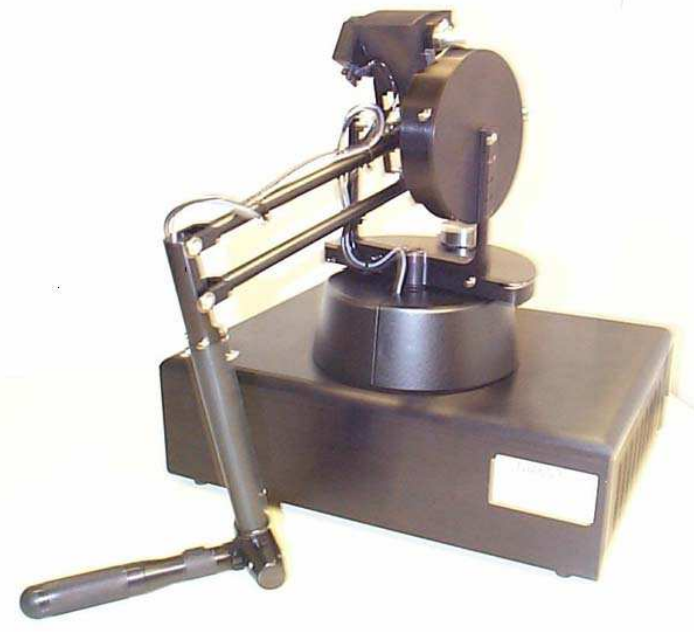

Figure 1: PHANToM haptic device

velocity of the stylus and the reference desired position and velocity.

The force fed back to the user is

$$
F=k_{z} e_{z}+\left(1+k_{g}\left|e_{z}\right|\right)\left(k_{p} e_{p}+k_{v} e_{v}\right)
$$

The first term enforces a preferred plane for the moves, and the second term is the PD feedback, with gains increasing with distance from the preferred plane. The justification for increasing the gains with distance from the preferred plane is addressed in section 3. Feygin et al. used a similar PD controller to train subjects in a gesture learning task [3].

The haptic dance, like real dance, has syntactic content. Moves are selected from a set of four moves: two clockwise circles, two counterclockwise circles, four upper half circles, and four lower half circles, all of which are executed in the frontal plane. The moves are illustrated in Figure 2.

The dance moves last eight beats and are performed to a soundtrack of a well-known song at 120 beats per minute. Each move takes four seconds. Perceptuomotor delays are known to be at or below about $350 \mathrm{~ms}$, so that this dance occurs slowly enough that it is possible for the subject to be replaying an open loop motion program through most of the duration of each move. Which move will follow the current move is not signalled in any way before the new move happens, although the timing of a switch is evident from the soundtrack. Then, for approximately 100 to $350 \mathrm{~ms}$ after a move transition, the subject may be doing one of two things:

- playing a motion program (alternately, predicting a zero-delay version of the input) that he has selected without knowing whether it corresponds to the correct movement, or

- reacting passively through the prior settings of impedance parameters on his limb and only actively 
1. two clockwise circles

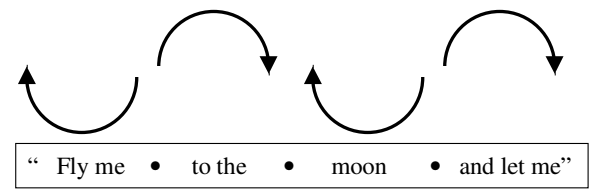

2. two counter-clockwise circles

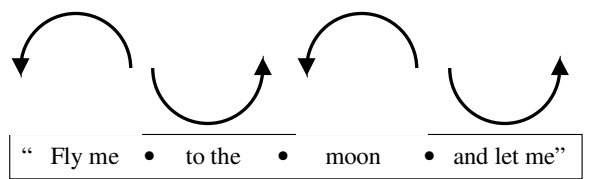

3. four upper half circles

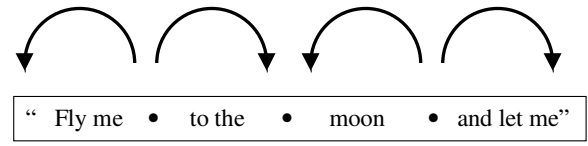

4. four lower half circles

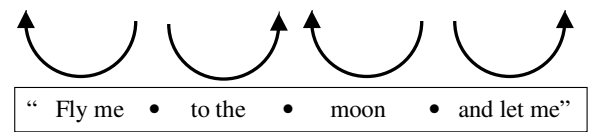

Figure 2: Dance moves

on actual sensed input (after a minimum neuromuscular delay).

If concurrent input prediction is indeed part of the subject's response, the input predicted does not necessarily belong to the set of actual input patterns.

\subsection{Protocol}

Five volunteer subjects familiar with the PHANToM were instructed to try to follow the PHANToM's lead. They were told that the dance was synchronized with the music and consisted of four simple circular moves. The subjects first trained for 60 seconds each on the four moves from figure 2. The subjects then trained for 90 seconds each on four two-move fixed sequences, such as two clockwise circles followed by four lower half circles.

The subjects then followed randomly generated move sequences lasting 120 seconds. Participants expressed varying levels of entertainment and frustration in trying to follow the moves.

\section{Metrics and Results}

In pursuit tracking experiments with pseudorandom smooth inputs, researchers have used metrics such as time to task completion, where the task is to move a pointer to within a certain tolerance of zero for 20 seconds, or total mean squared error over the task [2]. For the dance sequence following task, these metrics are inappropriate. Mean squared tracking error sums two sources of error that require separation: the move confusion error and the trajectory error for executing a move after, or in the case that, the move type is known. Another possible metric, time to task completion where the task is to infer the correct move transition, is not easily defined.

\subsection{Force tracking error}

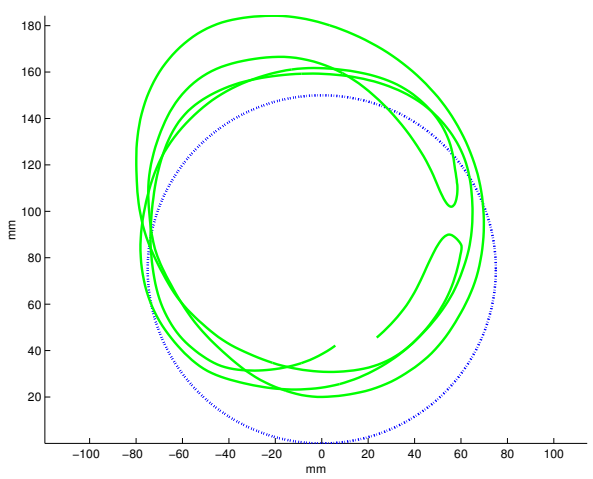

Figure 3: Position and target, $x y$

Figure 3 shows a typical human-PHANToM system trajectory for a sequence of about eight seconds, where the solid line is actual position. Note the vertical and slighter horizontal offset of the executed pattern from the target. Because the feedback gain for the position was relatively small compared to that for velocity (in [3] $k_{p}$ was about 200 times larger than $k_{v}$, where here $k_{p}$ is only twice as large as $k_{v}$ ), detecting these position offsets was difficult for subjects. Absolute position error is not a relevant metric. Instead, because force exerted by the PHANToM was the only display of error, force tracking is a more reasonable performance measure. Figure 4 shows PHANToM force output in the $y$ direction, averaged over four subjects and over ten instances each, during the 90 second practice sessions that repeated series of two moves. During these practice sessions, then, all of the moves and transitions should have been anticipated. It does appear that prior to direction reversals, subjects move in ways that modulate the force in the opposite direction to the imminent change.

Note that the output force is not the same as the force experienced by the user since the stylus is moving. The PHANToM does not sense user interaction forces, but could be instrumented to do so.

\subsection{Delays}

Both at move transitions and within moves, sudden direction reversals occur, so the time between reference 

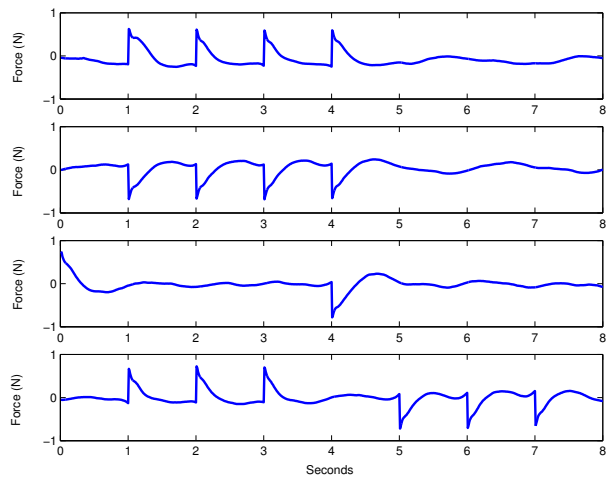

Figure 4: Forces in $y$, practice sessions with moves: 3 then 2, 4 then 1, 2 then 1, 3 then 4. See Figure 2.

position reversal and actual direction reversal might be defined as the time to task completion. This metric is called delay within this section.

In figure 5, the distribution (mean, quartiles, and outliers) of a typical subject's within move and at move transition reversal delays, with 53 data points for each category, is shown. Recall that a reversal within a move should be wholly predictable if subjects learn entire moves as precognitive trajectories. A reversal between moves, however, will happen in only about half of these between-move occasions and is unpredictable. The data suggest that shorter delays may be possible when the reversal is predictable. However, there is no statistically significant difference between the means of the within move and at move transition delays. Subjects may have occasionally predicted and planned for the reversal but in many instances fallen back to closed loop response, even with predictable reversals, because they were still novices at the dance.

Direction reversals requires sudden phase shifts of $\pi$ radians, and inertia prevents exact tracking of jump phase shifts, even if they are perfectly predicted. Perhaps the delay metric fails to meaningfully separate predictable and unpredictable reversals because the inertiamandated delay is close to the closed loop response

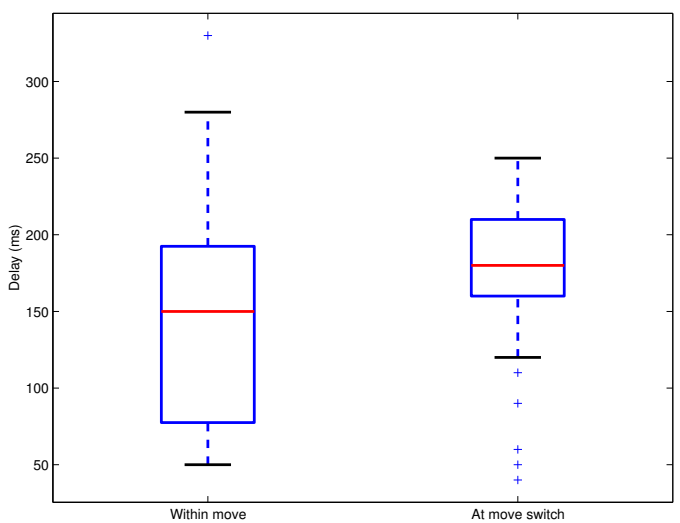

Figure 5: Median, quartiles, and outliers of delay

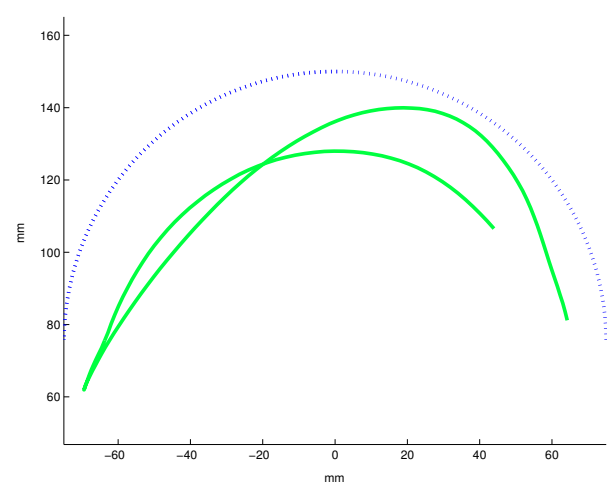

Figure 6: Desired reversal

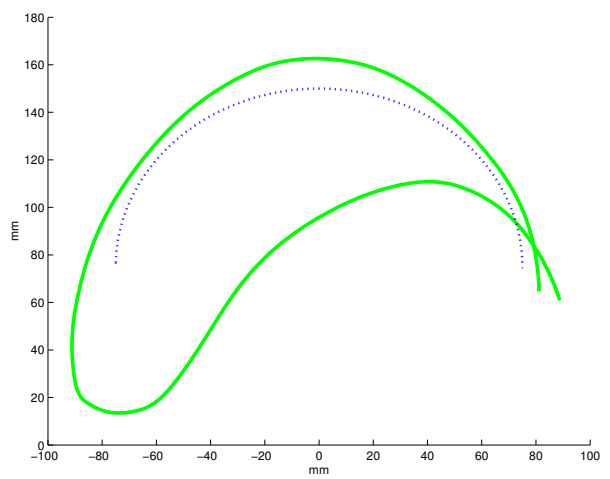

Figure 7: Mistakenly anticipated no reversal

delay. Also, all of the reversals may be predictable in a sense because they all fall on even beats of the music. If direction reversals can be prepared for, say, by trying to reduce the hand's effective mass, then subjects could prepare for even 'unpredictable' reversals, in which reversals and non-reversals are equally likely. Young noted that the most rapid adaptation to control system changes is achieved when subjects know both the pre-change and post-change systems well and where the transitions are accompanied by an auditory signal [9], and both of these conditions are met in this case.

\subsection{Large errors}

If a follower believed the move to come would be 1 but in fact it was 2 , the resulting error should be distinguished from simple tracking error. Move misclassifications were clearly recognizable in our data. Examples of a desired response and an obvious misclassification in this experiment's data are shown in figures 6 and 7 . Still, defining a large error at any reversal as an error in phase with square larger than twice the subject's mean squared error, the difference between the number of large errors at predictable reversals and unpredictable reversals did not rise to the level of statistical significance. 


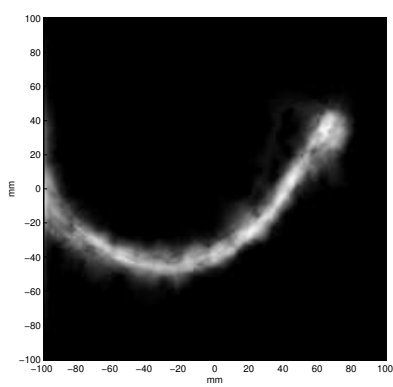

Figure 8: One subject's predictable reversals

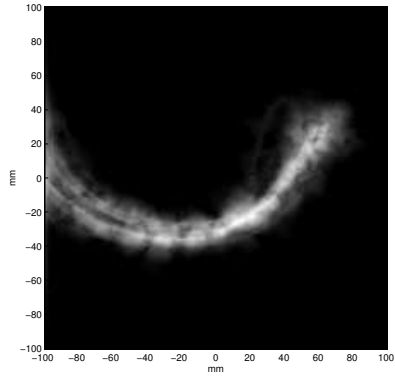

Figure 10: Second subject's predictable reversals

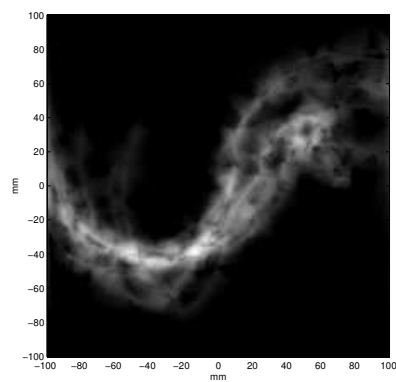

Figure 9: Same subject's unpredictable reversals

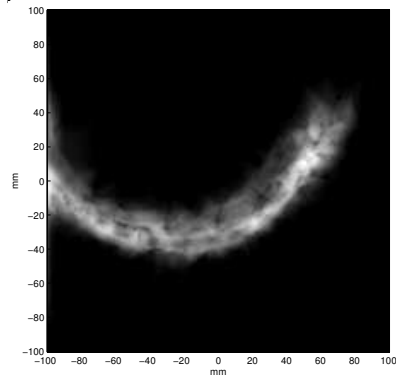

Figure 11: Same subject's unpredictable reversals

\subsection{Variance}

If subjects are using precognitive tracking in predictable reversals, but can not use precognitive tracking in unpredictable reversals, one would expect to see greater variance in performance at unpredictable reversals. The move density plots in Figures 8-11 each show 20 position traces recorded over $500 \mathrm{~ms}$ intervals after reversals, with image intensity related to the density of points near that location, i.e., brighter where movements coincide or nearly coincide.

The individual in figures 8 and 9 is also the individual with the highest mean phase errors of the five participants, and the distinction between the two plots is obvious to the eye. The individual in figures 10 and 11 had smaller mean phase error and less obvious distinction between the two plots. The remainder of the individual plot pairs were between these extremes.

The latter individual shown also clearly had a different, flatter half-circle in mind for the move than the former. This intra-subject variation with relative intersubject consistency should be noted.

\subsection{Connection in $z$ direction}

The $k_{g}$ term increasing the feedback gains is intended to mimic the feel of connection in dance. In partner dancing, the partners' hands remain in contact throughout their motion, even though grasping is not permitted, because each partner imposes a force on the other's hand in opposite directions. See figure 12. An

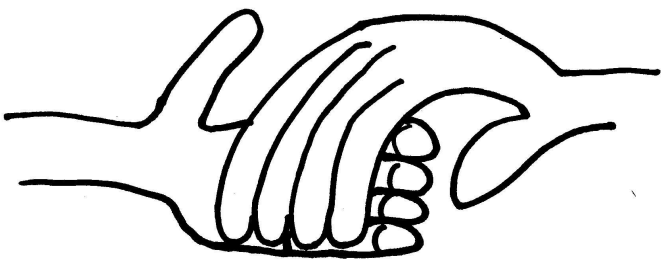

Figure 12: Connection: contact without grasping

experimental study of a similar type of non-grasping human movement, picking up and moving a box by pressing with an open palm on either side of it, is detailed in Zefran [10]. One common connection exercise for partner dancers is for each of them to hold one end of a piece of cloth, and to keep it taut throughout their dance so that the follower can feel small hand movements of the leader which would be imperceptible if the cloth were not taut.

The parallel to dance connection in the PHANToM game is the subject pulling back or pushing in the $z$ direction away from the target plane. The $k_{g}$ term in (1) was intended to encourage subjects to consistently pull away from the target plane in order to get higher error feedback. If subjects pulled with a constant force in the plane perpendicular to the pattern plane, a higher stiffness of the working arm would result. A stiffer working arm would require a more active response to the PHANToM's lead, reducing the incidence of extremely passive following. The PHANToM imposes only small forces, so following the dance requires active participation.

However, no consistent use of the $z$ plane increase in $k_{g}$ was observed. The $z$ value tends to oscillate with a frequency unrelated to the $x y$ plane error and position. Subjects did not regulate $z$ position actively, perhaps because grasping the PHANToM stylus is permitted. Subjects did not therefore need dance-type connection to maintain contact with the PHANToM. If grasping the stylus had been impossible, subjects may have pulled or pushed in the $z$ direction to maintain contact.

\section{Conclusions}

Humans can interact with a haptic device using the dancing metaphor, with a vocabulary of known moves sequenced unpredictably and a musical soundtrack to align timing. Intra-subject large variability contrasts with inter-subject strong consistency, which may indicate that subjects followed the moves based on their internal, precognitive models of the moves rather than in closed loop or pursuit tracking fashion. Density plots of the data illustrate that subjects responded differently when presented with predictable versus unpredictable 
direction reversals.

By fixing a very simple leader, we have demonstrated the power of the follower's vocabulary of moves to produce consistent behavior when the follower knows the choice of move. Going farther, a dual PHANToM two user system in development promises a more complete picture of the interplay between leader and follower, including the pre-transition signaling that enables followers to decide correctly which move will be next, and the leader's response to recognized move confusion in the follower with stronger leads or a move change. Beyond explaining the successful haptic coordination between dancers, the insights gained would inspire designs for future human-robot and even robot-robot interaction.

\section{Acknowledgements}

The authors gratefully acknowledge the support of the Science Foundation Ireland grant 00/PI.1/C067. SG was a visiting scientist at the Hamilton Institute while developing the research presented, and is also thankful for the support of a U. S. Department of Energy Computational Science Graduate Fellowship. RM-S is also grateful for support from EPSRC grant GR/R98105/01. I. Oakley, S. Wall, L. Brown, A. Crossan and S. Brewster provided valuable assistance with the experiments.

\section{References}

[1] S. Allin, Y. Matsuoka, and R. Klatzky, "Measuring just noticeable differences for haptic force feedback: implications for rehabilitation," in IEEE Haptics, March 2002, pp. 299-302.

[2] E.C.Poulton, Tracking Skill and Manual Control. Academic Press, 1974.

[3] D. Feygin, M. Keehner, and F. Tendick, "Haptic guidance: Experimental evaluation of a haptic training method for a perceptual motor skill," in IEEE Haptic Interfaces for Virtual Environment and Teleoperator Systems, March 2002, pp. 40-47.

[4] S. Gentry, S. Wall, I. Oakley, and R. MurraySmith, "Got rhythm? Haptic-only lead and follow dancing," in Proceedings of Eurohaptics Conference, Dublin, July 2003, pp. 481-488, at http://www.des.gla.ac.uk/ rod/publications/ GenWalOakMur.pdf

[5] R. Hess, The Control Handbook. CRC Press, 1996, ch. Human-in-the-loop control, pp. 1497-1505.

[6] R. J. Jagacinski and S. Hah, "Progressionregression effects in tracking repeated patterns,"
Journal of Experimental Psychology: Human Perception and Performance, vol. 14, no. 1, pp. 77-88, 1988.

[7] R. Magdaleno, H. Jex, and W. Johnson, "Tracking quasi-predictable displays," in Fifth annual NASA University Conference on Manual Control, 1969, pp. 391-428.

[8] I. Oakley, S. Brewster, and P. Gray, "Haptic gesturing," 2001, University of Glasgow technical report.

[9] L. Young, "On adaptive manual control," IEEE Transactions on man-machine systems, vol. 10, no. 4, pp. 292-331, 1969.

[10] M. Zefran, V. Kumar, G. Garvin, and J. Desai, "Experimental investigation of human two-arm manipulation," International conference on Mechanics in Medicine and Biology, 1996. 